\title{
Ehen (pl. Ihanan)
}

(voir Adebni (E.B. II, A53, p. 119-135), Antennes (E.B. V, A233, p. 710-712), Autels (E.B. VIII, A326, p. 1 170-1 175)

\section{H. Claudot-Hawad et G. Camps}

\section{OpenEdition}

Journals

Édition électronique

URL : http://journals.openedition.org/encyclopedieberbere/2131

DOI : $10.4000 /$ encyclopedieberbere. 2131

ISSN : 2262-7197

Éditeur

Peeters Publishers

\section{Édition imprimée}

Date de publication : 1 août 1996

Pagination : 2591-2595

ISBN : 2-85744-872-4

ISSN : $1015-7344$

Référence électronique

H. Claudot-Hawad et G. Camps, «Ehen (pl. Ihanan) », Encyclopédie berbère [En ligne], 17| 1996,

document E09, mis en ligne le 01 juin 2011, consulté le 25 septembre 2020. URL : http://

journals.openedition.org/encyclopedieberbere/2131; DOI : https://doi.org/10.4000/ encyclopedieberbere. 2131

Ce document a été généré automatiquement le 25 septembre 2020.

(c) Tous droits réservés 


\section{Ehen (pl. Ihanan)}

(voir Adebni (E.B. II, A53, p. 119-135), Antennes (E.B. V, A233, p. 710-712), Autels (E.B. VIII, A326, p. 1 170-1 175)

\section{H. Claudot-Hawad et G. Camps}

\section{Ehen, terme touareg (H. Claudot-Hawad)}

1 Ce terme touareg pourrait être traduit en français par l'appellation de «maison » qui sert à désigner à la fois l'habitation (sans préjuger du type de logement qu'elle constitue), son aménagement intérieur, les personnes qui y vivent et enfin la descendance ou la lignée d'un individu.

2 En touareg, éhen renvoie bien sûr à la tente* en cuir ou en natte qui forme l'habitation la plus courante chez les nomades, mais s'applique également aux autres formes d'habitat lorsque le discours fait référence au « chez-soi ». 


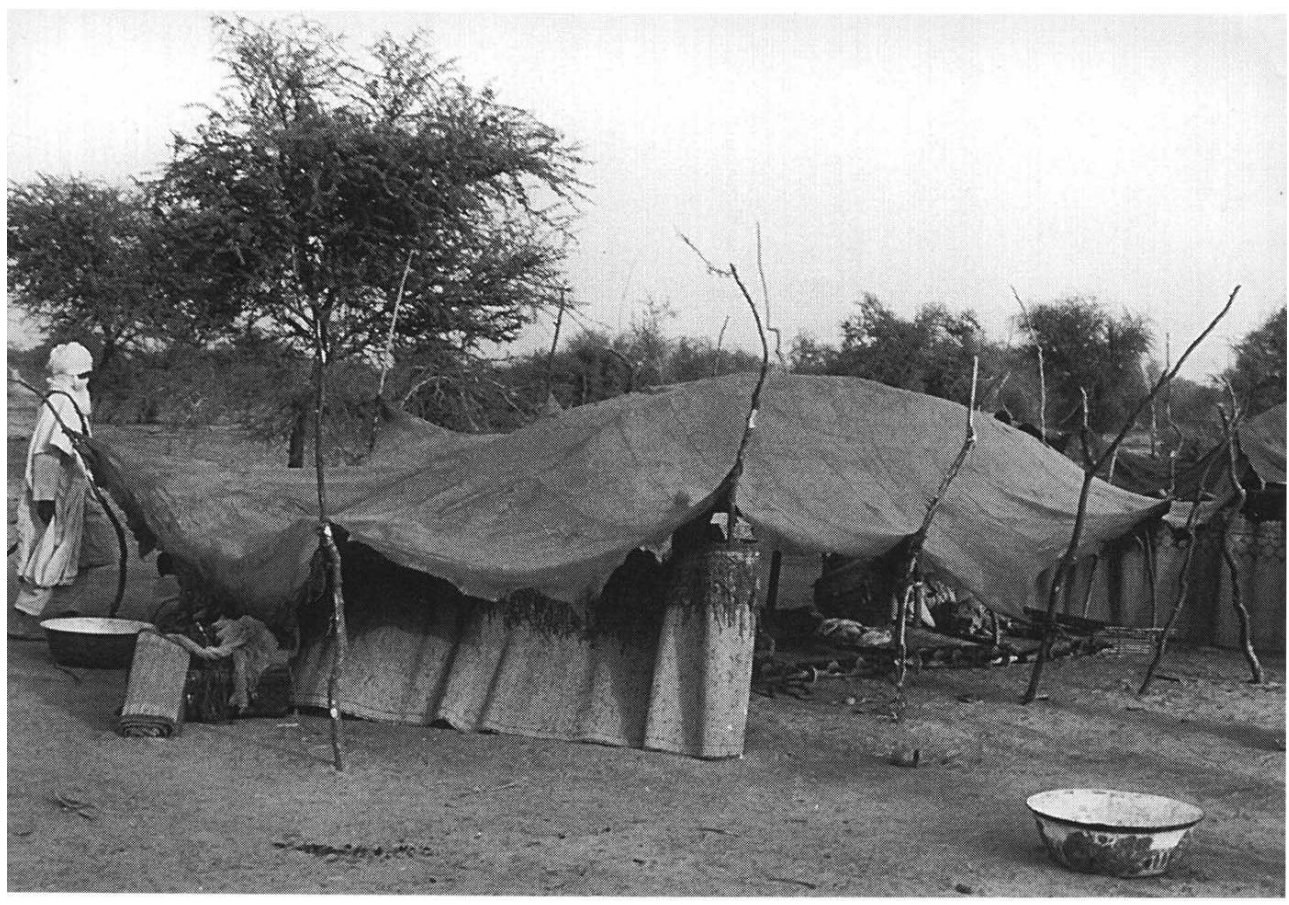

Pour un nomade, le cœur de l'espace habité et civilisé par opposition au monde non domestiqué et sauvage qu'il aura à affronter est d'abord sa tente, c'est-à-dire la tente où il il est né, celle de sa mère. En effet, dans la plupart des groupes touaregs, la règle de filiation est matrilinéaire et les enfants sont rattachés à la lignée de leur mère. Dans ce contexte, la tente est un bien féminin, constitué au moment du mariage. Chaque nouvelle tente doit comprendre au moins quelques éléments de la tente maternelle. De cette manière se trouve prolongé symboliquement l'abri originel qui permit d'« être » aux ancêtres de la famille car, suivant la cosmogonie touarègue*, aucun être, aucun objet ne saurait exister sans la protection d'un abri.

Le terme éhen s'utilise également pour désigner métaphoriquement l'«épouse ", détentrice de la tente qui abrite le couple. Refuge temporaire pour l'homme cette fois puisqu'en cas de divorce ou de veuvage, il perdra simultanément épouse et logis. Au contraire, la femme ne « sort » jamais de l'abri ; elle se confond quelquefois avec lui. Et si elle se marie à l'extérieur de son clan (appelé tawsit et conçu comme un groupe de descendance unilinéaire), elle va créer une véritable enclave territoriale dans le campement de son mari en y installant sa tente qui, certes, lui appartient en propre mais représente également sa lignée toute entière.

5 L'un des principes chers à la société touarègue est celui de l'autonomie économique de la femme qui, en général (à l'exception des suzerains Iwellemmeden), arrive chez ses alliés munie non seulement de sa tente et de tous les ustensiles domestiques nécessaires, mais aussi de troupeaux, de biens, de serviteurs en nombre suffisant pour la faire vivre tout à fait indépendamment de son époux si elle le désire (Claudot-Hawad et Hawad, 1987 ; Worley, 1991). L'indépendance de cette femme par rapport à ses alliés garantit en même temps l'indépendance de la lignée qu'elle représente.

6 Enfin, éhen désigne la famille utérine descendant de cette ancêtre «mère » qui fonda l'abri et assura ainsi le devenir de sa lignée. Le nom de l'ancêtre légendaire des Kel Ahaggar, Ti-n-Hinan, est dans ce sens souvent interprété comme une altération de 
l'expression poétique de ti n (i)hanan, qui signifie littéralement « celles des tentes » et sert à désigner les femmes du campement, c'est-à-dire le cœur de la matrilignée.

Éhen s'applique plus particulièrement à l'ensemble des femmes qui constituent ce noyau matriliénaire de la parenté, axe suivant lequel sont transmis des droits ou des biens inaliénables qui, de la même façon que l'abri, vont assurer la survie de la communauté. Le terme équivaut également dans ce sens à ébawél ou ébategh, qui peuvent dénommer l'abri que constitue la famille utérine, s'appliquant aussi à l'ancêtre féminine matrilinéaire et enfin à la tente qui, transmise de mère en fille, marque la continuité de la famille.

8 En fait, chaque fois qu'une fille de la parenté se marie, c'est-à-dire chaque fois qu'une nouvelle tente s'établit, une part des biens de la « grande tente » ou «tente mère » lui est attribuée pour qu'elle puisse survivre. Mais cette cession est temporaire, car le jour où le mariage est dénoué par divorce ou veuvage, la femme, sa tente et les biens qui lui permettent de jouer le rôle d'abri, reviendront à leur point de départ, devenant un abri dans l'abri (H. Claudot-Hawad et Hawad, 1987).

Ainsi, la société peut être vue comme un emboîtement infini de tentes où, à chaque palier, l'â̂né(e) du cercle de parenté est censé jouer le rôle du pilier central qui soutient le velum, entouré et assisté par les piquets latéraux. Comme les unités sociales qui la composent également, la société toute entière peut être dénommée éhen $n m a$, «tente de la mère" tandis que les relations de rivalité entre pairs, qui caractérisent les membres des tentes de rang égal, se rendent dans le sud-ouest du pays touareg par le terme ehennemu (correspondant ailleurs à tamanjaq ou tamañhaq), mot qui serait composé à partir de éhen n maw, "tente des mères ». Cette grille de lecture s'applique également, dans son acception pyramidale, aux relations qui lient suzerains et tributaires et se projette dans la distribution des différents groupements sur le territoire politique des confédérations, considéré comme bâti à l'image d'une tente (Claudot-Hawad et Hawad, 1986).

Structure de la tente touarègue d'après $M$. Reygasse

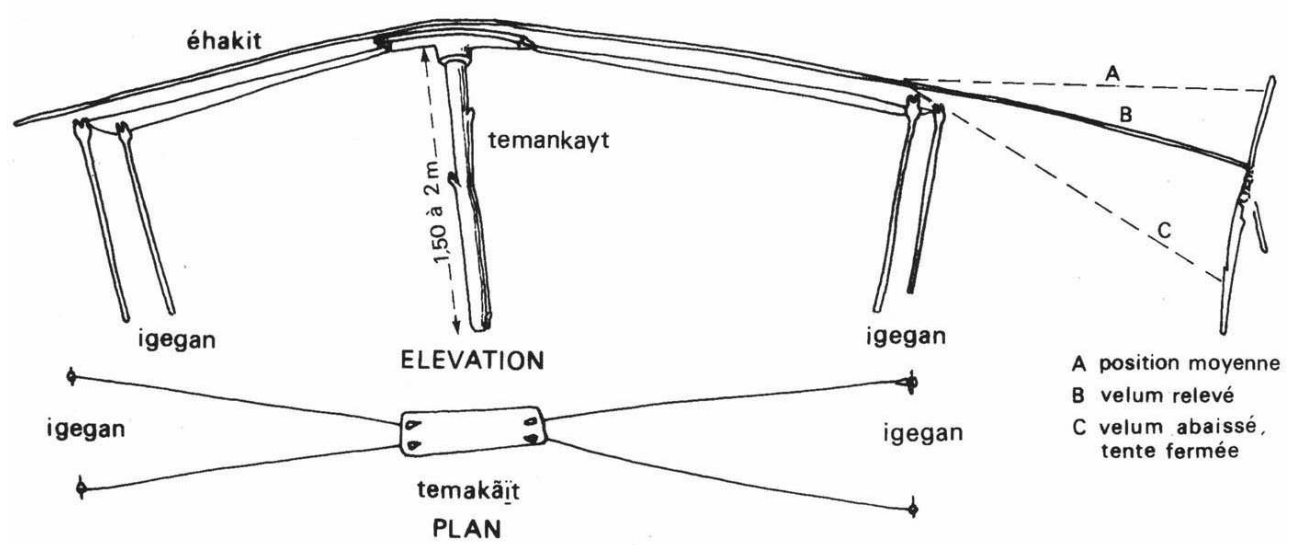


Tente de nattes de l'Aïr. Photo H. Claudot-Hawad

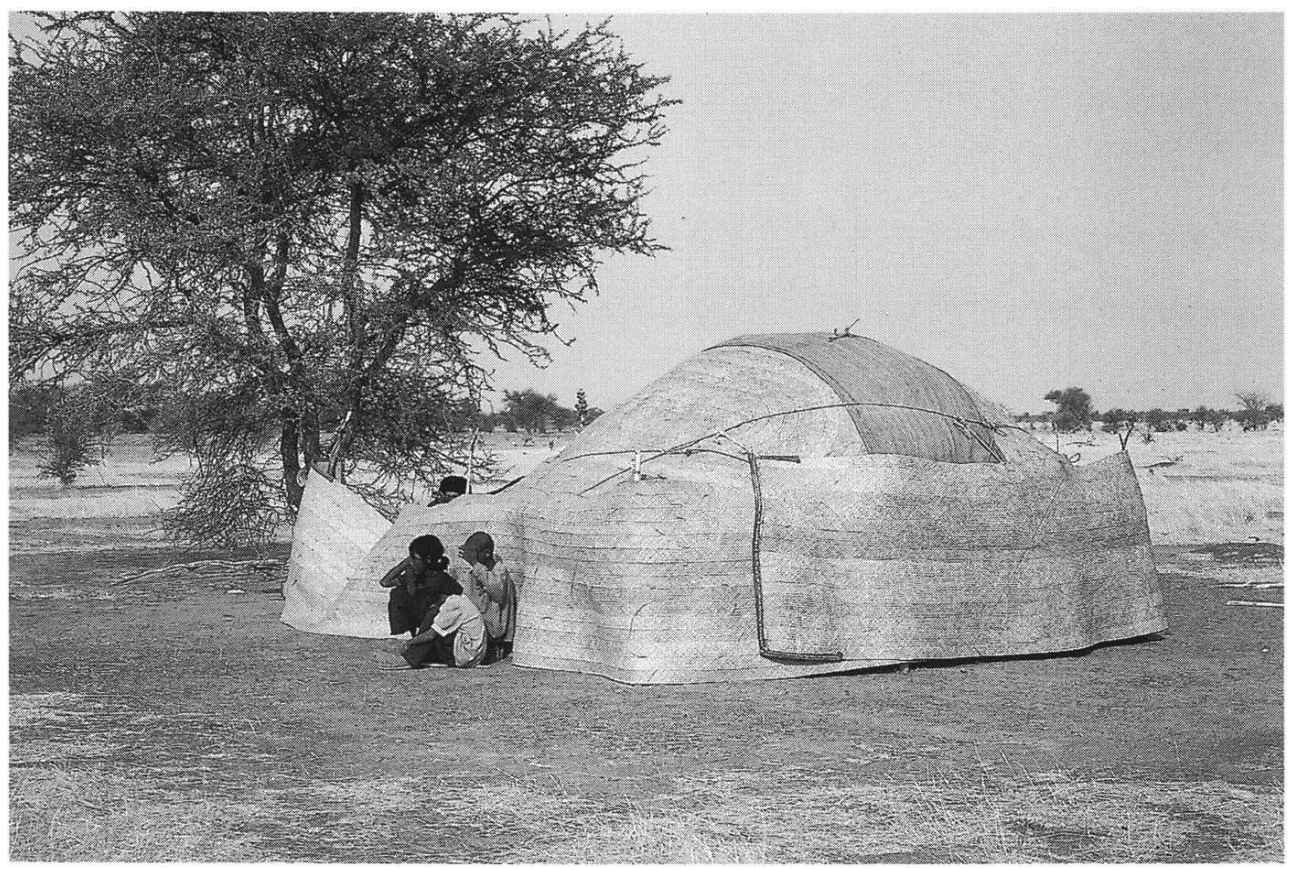

\section{Ehen n-Fatima («Tente de Fatima ») (G. Camps)}

Les Touaregs de l'Ahaggar désignent par cette expression une variété assez commune de monuments en pierres sèches que les archéologues ont nommée « monument en fer à cheval » bien que leur plan ne dessine pas toujours cette figure. En fait, il existe deux sortes d'ehen n-Fatima. Dans la première le corps principal décrit un demi ou trois-quart de cercle; l'interruption étant située dans le secteur est. C'est en face de cette ouverture que se dresse une ligne d'autels* turriformes en pierres sèches identiques à ceux qui accompagnent certains idebnan (voir adebni*). 
Différents types de « Ehen n-Fatima », d'après le Dr Pervès
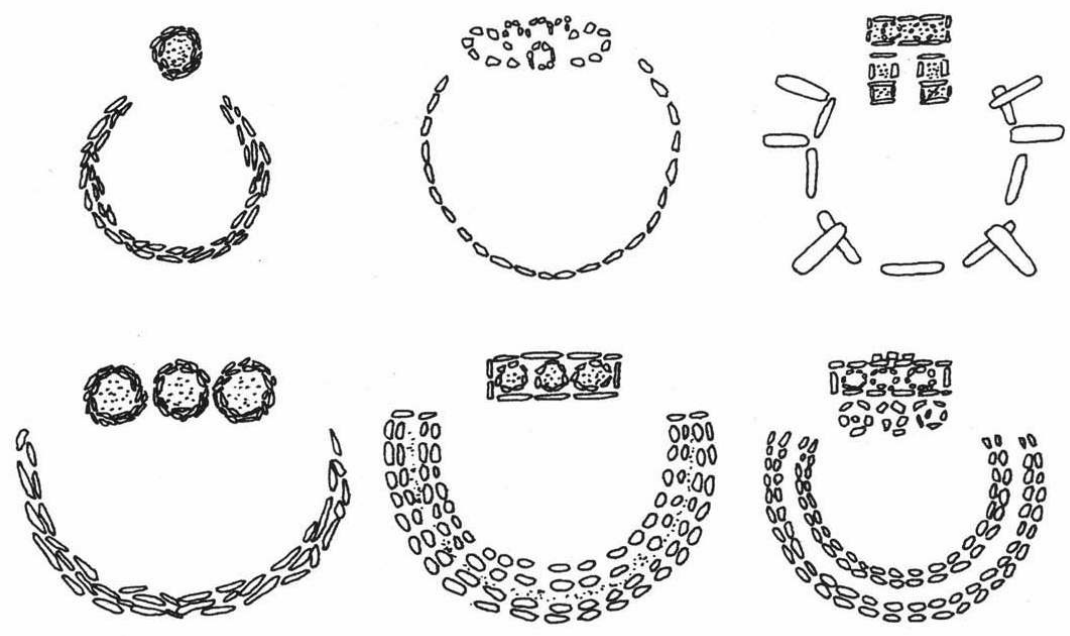

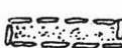
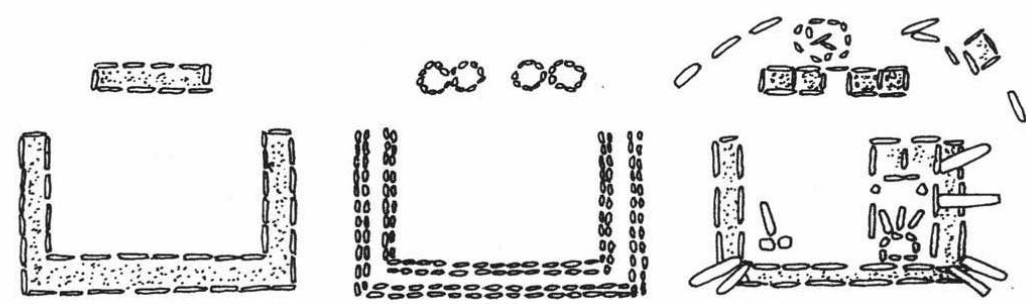

«Tentes de Fatima » à éléments coudés de l'oued Ouahen à Tiffert (Ahaggar), relevé G. Camps
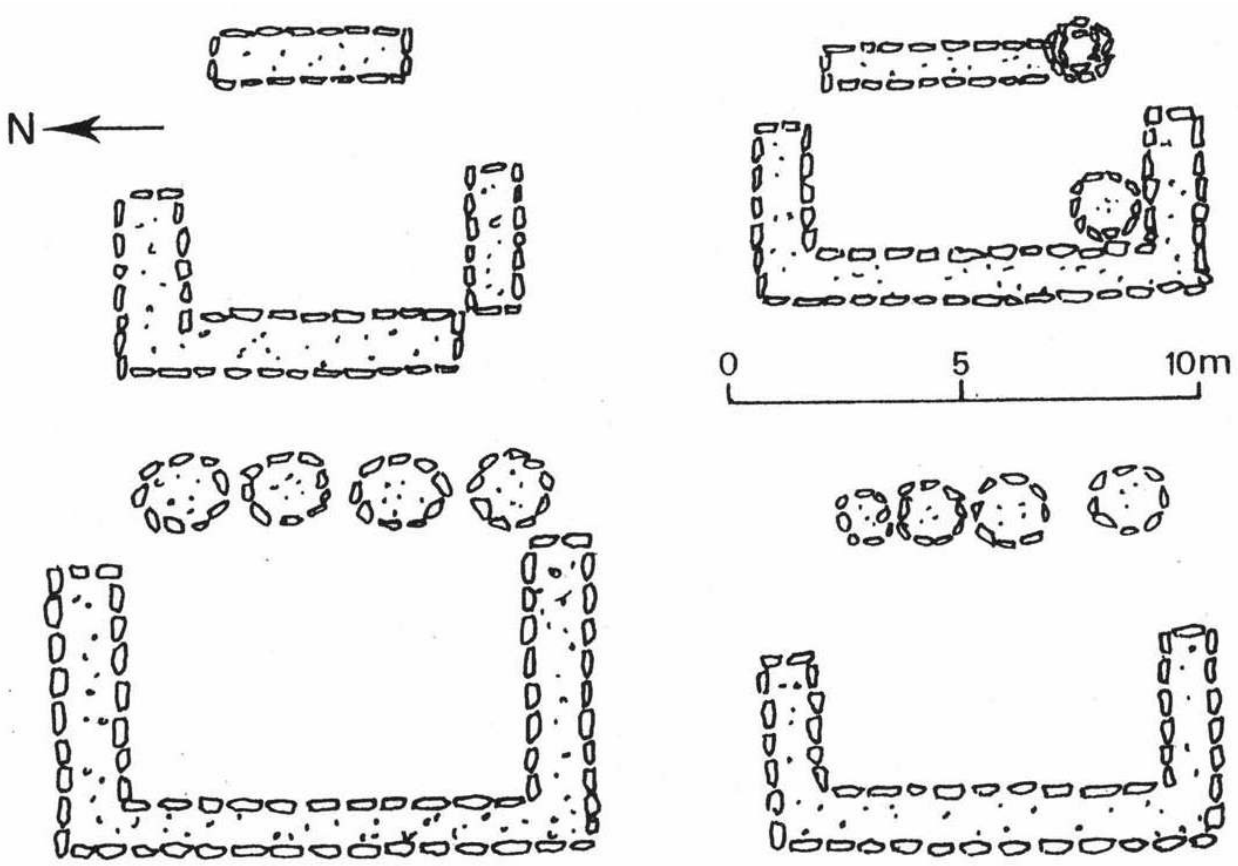

11 Il est exceptionnel qu'il n'y ait qu'un seul autel : dans la majorité des cas, ces annexes sont au nombre de quatre. L'autre type de «tente de Fatima » est constitué d'un corps coudé à angle droit sur trois côtés, également ouvert à l'est et cette ouverture est soulignée, comme dans le premier type, par une ligne d'autels ou par une annexe rectangulaire qui peut enclore des autels. Seul le premier type de «tente de Fatima» 
mérite le nom de « fer à cheval »; aussi pour éviter toute confusion, il serait préférable de bannir cette dénomination ou, si on la conserve pour le premier type, de réserver au deuxième type le nom de monument coudé, Ehen n-Fatima étant le nom général réservé à ces deux variétés de monuments très proches l'une de l'autre. Il importe de remarquer, toutefois, que ces deux types, de conception identique, ne connaissent pas de forme intermédiaire et sont exclusifs l'un de l'autre ; à ma connaissance, ces deux variétés ne sont jamais associées sur le même site.

Les ehen n-Fatima sont généralement isolés, mais on les trouve parfois associés par paire. Dans l'oued Ouahin (région de Tiffert, Ahaggar), à $80 \mathrm{~m}$ de distance subsistent deux paires de ces monuments coudés. Deux d'entre eux présentent une anomalie ; l'un a subi une interruption de la construction à l'angle sud et sur un autre un seul autel fut élevé mais il est situé à l'extrémité nord d'une annexe rectangulaire qui généralement remplace les autels.

L'architecture de ces monuments est très simple. Ils ne présentent qu'une très faible élévation et sont plus dessinés sur le sol que véritablement construits. Suivant les régions, ils sont constitués d'un double cordon de galets ou de fragments de dalles de basalte plantés en deux lignes sur le sol ; dans les deux cas un remplissage de gravier ou de petites pierres et de sable a été versé entre les parois.

14 Les rares fouilles pratiquées dans ces monuments (Reygasse à Abalessa, G. Camps à In Eker) n'ont donné aucun résultat.

15 Les «Tentes de Fatima » sont assez fréquentes dans l'Ahaggar et ses annexes (Tassili n'Ajjer, Tidikelt, Adrar Ahnet, Adrar Tiouiyne...), ces monuments paraissent plus rares dans les autres massifs du Sahara central et méridional (Aïr, Adrar des Ifoghas) ; il n'en a pas été signalé au Tibesti ni en Mauritanie. Leur aire de répartition correspond assez exactement à celle des groupes touaregs actuels.

16 Certainement antéislamiques (malgré l'allusion à la fille du Prophète), ces monuments ne sont pas oblitérés par d'autres comme cela arrive assez fréquemment chez ceux « en trou de serrure », ainsi que les croissants et certaines bazinas, aussi ne doivent-ils pas remonter à des temps très reculés.

\section{BIBLIOGRAPHIE}

\section{H. Claudot-Hawad}

Badi D., « Tin-Hinan : un modèle structural de la société touarègue », Études et Doc. Berbères, t. 12, 1994, p. 199-205.

Bernus S., Bonte P., Brock L., Claudot H., (éds.), Le fils et le neveu, Jeux et enjeux de la parentée touarègue, Cambridge Uni Press/MSH, 1986.

Casajus A., La tente dans la solitude, La société et les morts chez les Touaregs Kel Ferwan, MSH, Paris, 1987. 
Claudot-Hawad H., et Hawad M., « La conquête du vide ou la nécessité d'être nomade », Revue de l'Occident musulman et de la Méditerranée n 41-42, 1986, p. 397-412. Id., « Le lait nourricier de la société ou la prolongation de soi chez les Touaregs ", in Hériter en pays musulman (Gast, éd.), CNRS, Paris, 1987, p. 129-156.

Claudot-Hawad H., Les Touaregs, Portraits en fragments, Édisud, 1993, 213 p.

Foucauld Ch. de, Calassanti-Motylinski A. de, Textes touaregs en prose, Éd. critique avec traduction par S. Chaker, H. Claudot et M. Gast, Édisud, 1984, textes 1 et 2.

Lhote H., Cornent campent les Touaregs, J. Susse, Paris, 1947, 270 p.

Nicolas F., « Les industries de protection chez les Twareg de l'Azawagh », Hespéris, 1938, p. 1-42.

Worley B., Women's war drum, Women's wealth : The social construction of female autonomy and social prestige among Kel Fadey Twareg pastoral nomads, Ph. D. Thesis, Columbia Univ., New York, 1991, $482 \mathrm{p}$.

INDEX

Mots-clés : Artisanat, Ethnologie, Famille, Habitation, Nomadisme, Sahara, Touareg 